\title{
New Resistive Switching and Self-Regulating Heating in Foliated Graphite/Nickel Polyvinyl Chloride Nanocomposites
}

\author{
Omar A. Al-Hartomy, ${ }^{1,2}$ Falleh Al-Salamy, ${ }^{3}$ A. A. Al-Ghamdi, ${ }^{2}$ Attieh A. Al-Ghamdi, \\ A. M. Abdel Daiem, ${ }^{2,5}$ and Farid El-Tantawy \\ ${ }^{1}$ Department of Physics, Faculty of Science, University of Tabuk, Tabuk 71491, Saudi Arabia \\ ${ }^{2}$ Department of Physics, Faculty of Science, King Abdulaziz University, Jeddah, P.O. Box 80203, Jeddah 21569, Saudi Arabia \\ ${ }^{3}$ Department of Mathematics, Faculty of Science, University of Tabuk, Tabuk 71491, Saudi Arabia \\ ${ }^{4}$ Electronics, Communications, and Photonics Program, King Abdulaziz City for Science and Technology, Riyadh, Saudi Arabia \\ ${ }^{5}$ Department of Physics, Faculty of Science, Zagazig University, Zagazig 44519, Egypt \\ ${ }^{6}$ Department of Physics, Faculty of Science, Suez Canal University, Ismailia 41522, Egypt
}

Correspondence should be addressed to Farid El-Tantawy, faridtantawy@yahoo.com

Received 18 May 2011; Revised 26 June 2011; Accepted 18 July 2011

Academic Editor: Sadhan C. Jana

Copyright ( 2011 Omar A. Al-Hartomy et al. This is an open access article distributed under the Creative Commons Attribution License, which permits unrestricted use, distribution, and reproduction in any medium, provided the original work is properly cited.

\begin{abstract}
Polyvinyl chloride- (PVC-) based nanocomposites containing nanosized graphite and nickel nanoparticles (GN) as conductive fillers to achieve positive temperature coefficient of resistance (PTCR) thermistors and self-regulating heater function have been successfully fabricated. The microstructure of the foliated graphite and nanocomposites was examined by scanning electron microscopy (SEM). The effect of GN content on the static electrical conductivity, carrier's mobility, and number of charge carriers of the nanocomposites was studied. The applicability of nanocomposites as PTCR thermistors was examined by monitoring the conductivity as a function of temperature. It is found that the conduction mechanism in PVC/GN nanocomposites is governed by tunneling mechanism. Also, the applied voltage versus current and temperature were studied to check the applicability of composites as self-regulating heater. The results show that the PVC/GN nanocomposites might have potential applications in PTCR devices, self-regulating heater, and temperature sensors.
\end{abstract}

\section{Introduction}

The design and applications of electroactive conductive polymer nanocomposites in the electrical and electronic fields have significantly affected the modern technology and added a new dimension to scientific interest [1-3]. The most economical route for fabrication of electro active conducting polymer composites is the inclusion of a conductive filler such as carbon black, carbon nanotubes, graphite, metal powders, ceramic oxides, polyaniline, and others in an insulating polymer matrix and subsequent compaction by compression molding [4-8]. These electro active nanocomposites attracted great interests due to their potential applications in various hi-tech aspects, for example, positive and/or negative temperature coefficient of resistance (PTCR/NTCR) thermistors [9], electrochemical displayers
[10], sensors $[11,12]$, catalysis [13], redox capacitors [14], electromagnetic shielding [15, 16], radar evasion [17], rechargeable batteries $[18,19]$, conductive inks and antistatic textiles $[20,21]$, and aero space $[3,12]$ as well as in secondary battery and bipolar plates in the polymer electrolyte membrane fuel cell, and so forth [20]. A PTCR composite is a grain-boundary resistive effect which is characterized by an increase in resistivity with increasing temperature [14-16]. The traditionally thermistor materials irrespective of its applications can be broadly divided into three categories, namely, metals (such as titanium and platinum), semiconductors (such as $\mathrm{Si}, \mathrm{Ge}$, and $\mathrm{SiC}$ ), and ceramic oxide semiconductors (like single- and multicomponent oxides) $[17,18]$. The use of traditional thermistors has been confined because of the low room temperature conductivity and the oxidation of the metallic particles that severely limits 
the current flow $[11,19]$. Furthermore, when particulate fillers such as carbon black and metal are added to polymeric systems, a huge increase in the melt viscosity is observed which makes the melt processing of the composites more difficult and disrupts the mechanical properties restricting their application of thermistors [21]. Recently, the demand of PTCR composites with high room temperature conductivity is rising in markets, in a wide range of applications especially in automobiles that need PTCR materials with large current carrying capacity [14], self-regulating heater [18], protection circuits [19], temperature sensors [8, 15], infrared radiation detector sensors, flow meters [5], current limiters [7], temperature controlled heater [10], ambient thermal state indicators [11], and so on. To our best knowledge, there have been no reported investigation of the PTCR thermistors and self-regulating heater from dispersed dual conducting phases foliated graphite and nickel nanoparticles (GN) into polyvinyl chloride (PVC) matrix. In the current study, our investigations are focused on the fabrication and development of newly electrically conducting PVC-based composites, containing multicomponent fillers (i.e., GN) and on the possibilities for their potential application in PTCR thermistors devices and self-regulating heater.

\section{Experimental Details}

2.1. Materials. Natural flake graphite purchased from Shandong Qingdao Graphite Company (Qingdao, China) with an average diameter of $500 \mu \mathrm{m}$ is used for preparing the expanded graphite (exfoliated). Commercially concentrated sulfuric and nitric acids obtained from Egyptian Chemical Company, Cairo, Egypt, were used as chemical intercalate and oxidizer to prepare exfoliated graphite. $95 \%(\mathrm{v} / \mathrm{v})$ alcohol and distilled water were used as solvents for preparation of foliated (nanosheets) graphite. Polyvinyl chloride (PVC) with an average molar mass number being 2000 was supplied from Tokyo Chemical Industry Co., Ltd., Tokyo, Japan. Nickel powder was supplied by Wako Chemical Company with particle size of 10 micrometers.

2.2. Preparation of Exfoliated Graphite. Natural flake graphite was first dried in a vacuum oven for $24 \mathrm{~h}$ at $120^{\circ} \mathrm{C}$. Then, a mixture of concentrated sulfuric and nitric acids (ratio $3: 1$, $\mathrm{v} / \mathrm{v}$ ) was slowly added to a glass flask containing graphite flakes with vigorous stirring. After $36 \mathrm{~h}$ of reaction, the acid-treated graphite flake was filtered and washed with deionized water until the $\mathrm{pH}$ level of the solution reached 6.6. After being dried at $100^{\circ} \mathrm{C}$ for $72 \mathrm{~h}$, the resulting graphite intercalation compound was subjected to a thermal shock at $1050^{\circ} \mathrm{C}$ for 30 seconds in a muffle furnace to form exfoliated (expanded) graphite.

2.3. Preparation of Graphite Nanosheets. $1 \mathrm{~g}$ of exfoliated graphite was mixed and saturated with $400 \mathrm{~mL}$ alcohol solution consisting of alcohol and distilled water with a ratio of $68: 32$ for $24 \mathrm{~h}$. The mixture was then subjected to ultrasonic irradiation with a power of 400 watt for $24 \mathrm{~h}$. After $24 \mathrm{~h}$ of sonication, exfoliated graphite particles were effectively fragmented into foliated (nanosheets) graphite. The foliated graphite dispersion was then filtered and dried at $100^{\circ} \mathrm{C}$ to remove residual solvents. The as-prepared foliated graphite powder will be called graphite nanosheets $(G)$ throughout the paper.

2.4. Preparation of Nickel Nanoparticles. The starting material for ball milling is $\mathrm{Ni}$ powder $(250$ mesh) with purity above $99.9 \%$. The Ni powder was placed in a stainless steel vial with stainless steel balls of $10 \mathrm{~mm}$ diameter. The ball-topowder ratio 20:1 was used in the planetary mill (Marconi MA 350 ball mill) at $400 \mathrm{rpm}$ under argon atmosphere for $6 \mathrm{~h}$ to obtain nickel nanoparticles $(\mathrm{N})$.

\subsection{Preparation of Conducting PVC-Based Nanocomposites.} The conducting fillers are composed of graphite nanosheets (thickness: $30-50 \mathrm{~nm}$ ) with an average thickness of about $40 \mathrm{~nm}$ (the number of sheets in the platelets is 100) and $\mathrm{Ni}$ with an average particles size of $12 \mathrm{~nm}$. First, the as-prepared graphite nanosheets and nickel nanoparticles 80/20 wt\% were mixed together in a kitchen machine Philips mixer for $1 \mathrm{~h}$. Second the as-received conducting mixture was added to the PVC matrix and mixed for $30 \mathrm{~min}$. Then the mixtures were transferred to a hot press and tested samples were prepared at pressure of $40 \mathrm{KN} / \mathrm{m}^{2}$ and temperature of $180^{\circ} \mathrm{C}$ for $30 \mathrm{~min}$. Several batches of $\mathrm{PVC} / \mathrm{GN}$ weight ratios were considered: $97: 03,96: 07,91: 09$, and $88: 12$, respectively, and abbreviated as GN3, GN6, GN9, and GN12, respectively.

2.6. Characterization and Tests. Scanning Electron Microscopy (SEM) micrographs and energy dispersive X-ray analysis (EDX) spectra were obtained with a JEOL JSM 6400 scanning electron microscope equipped with a Link analytical system. The electron energy used was $15 \mathrm{keV}$. The specimens were coated with carbon using a vacuum evaporator (JEOL, GEE 500). The specific electrical conductivity temperature dependence was performed for the composites by dc two-probe method using a computer-aided system in the temperature range from $22^{\circ} \mathrm{C}$ to $150^{\circ} \mathrm{C}$ in air. The current was measured through the sheet sample under a steady constant voltage using a digital Keithley 642 electrometer. The two sides of the samples were bended with $\mathrm{Cu}$ rod during curing process to reduce the contact resistance. Hall effect measurement was used to determine the carrier type, concentration, and mobility. The measurements were performed using the van der Pauw configuration under direct current ranging from $2 \times 10^{-3}$ to $4 \times 10^{-4} \mathrm{~A}$, and the applied magnetic field was $0.33 \mathrm{~T}$. The equipment used for this purpose was a Keithley source meter (model 231). The current-voltage $(I-V)$ characteristic curves were measured with a precision semiconductor parameter analyser (Keithley 442 source measure unit). Silver paste was used to ensure a good contact of the sample surface with copper electrodes. The temperature for each applied voltage on the sample was measured by thermocouple embedded inside the samples during compression molding. The dielectric constant of the composite specimen was obtained from the capacitance of the specimen, the area of Ag electrode, and the thickness 




(a)

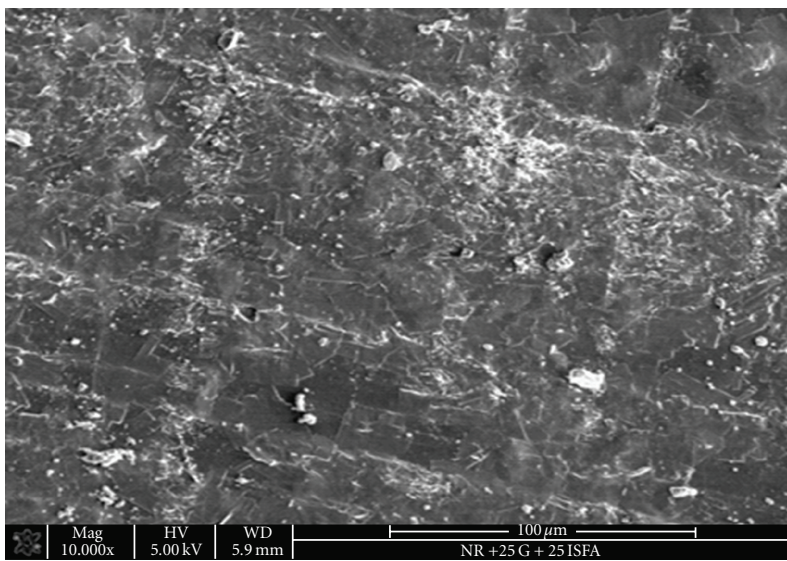

(c)

c)

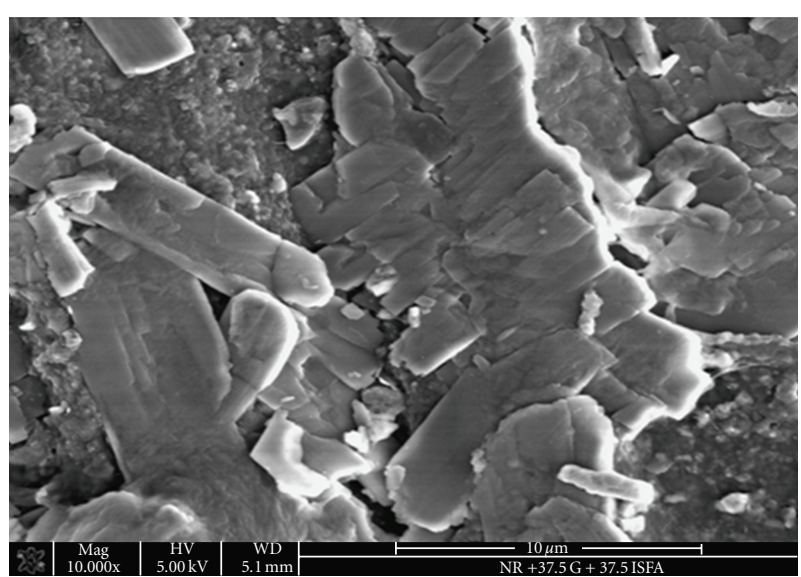

(b)

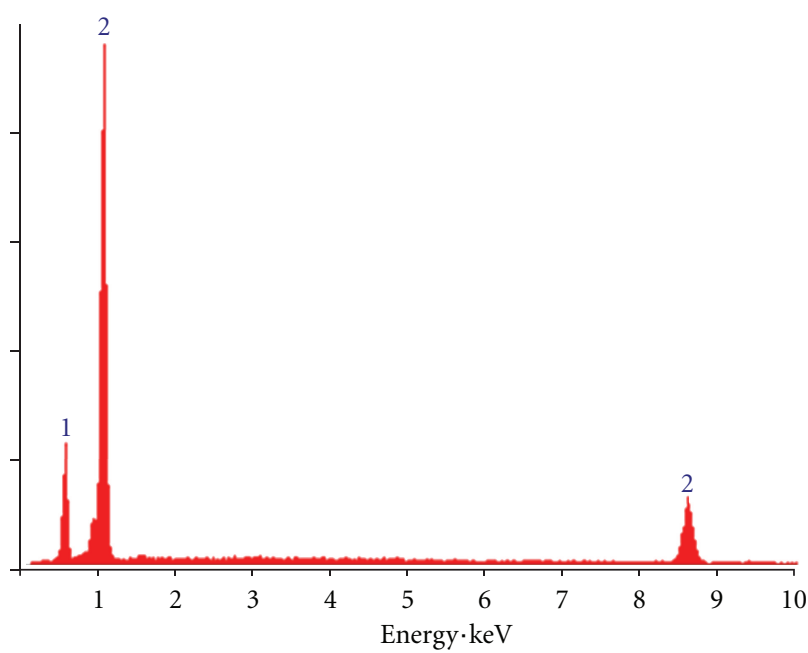

1: Nickel 2: Graphite

(d)

FIGURE 1: (a) SEM images of graphite nanosheets prepared after $24 \mathrm{~h}$ ultrasonic treatment and (b) SEM image of GN12 sample magnification with 10 x 0000. (c) SEM image of GN12 sample magnification with 1000 and (d) EDX pattern recorded for GN nanoparticles.

of the composite specimen. Circular-shaped samples with $20 \mathrm{~mm}$ diameter and ca. $1.3 \mathrm{~mm}$ thickness were prepared for dielectric measurement. Silver paste was used to coat the two sides of the specimen as electrodes. The capacitance of the specimen was measured with a network analyzer (E38362B) at frequency of $100 \mathrm{~Hz}$. The specific heat capacity $\left(C_{p}\right)$ of the samples was calculated using differential scanning calorimeter measurements (Perkin-Elmer DSC-2; Perkin Elmer Cetus Instruments, Norwalk, CT) using sapphire as the reference material.

\section{Results and Discussion}

3.1. Network Structure Observation of Nanocomposites. SEM observations from cross-sections of foliated graphite were performed to understand the microstructure occurrence during intercalation. Figure 1(a) shows the SEM image of foliated graphite nanosheets prepared based on $24 \mathrm{~h}$ ultrasonic irradiation. It is clearly apparent that the exfoliated graphite worms have been completely torn into foliated, named graphite nanosheets. The powder has an apparent density of about $0.015 \mathrm{~g} / \mathrm{cm}^{3}$, much lower than the mass density of the original natural flake graphite which is $2.2 \mathrm{~g} / \mathrm{cm}^{3}[1,7]$.

Generally, the entire concept of electrical properties like percolation and PTCR behavior in nanocomposite thermistors is mainly dependent on microstructure and filler aspect ratio $[5,21]$. The surface morphology of foliated graphite and the composites was examined by scanning electron microscopy. Typical SEM micrograph of GN12 with different magnification is depicted in Figures 1(b) and 1(c). As can be seen, the GN nanoparticles have good affinity and 


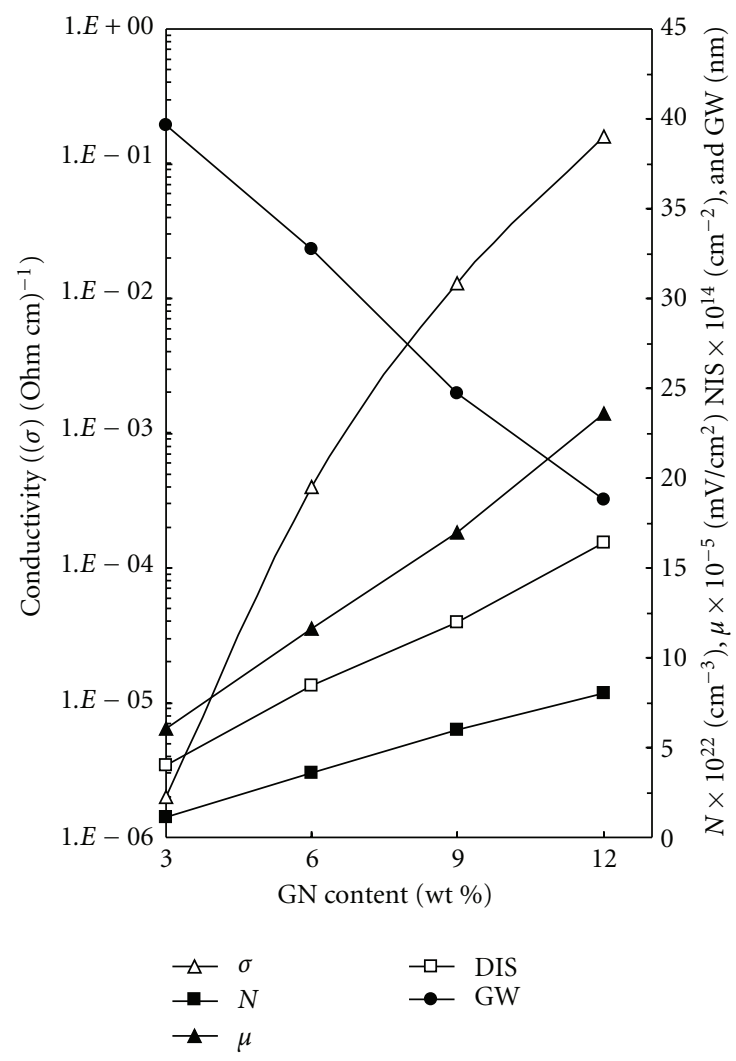

Figure 2: Electrical conductivity $(\sigma)$, carriers mobility $(\mu)$, and number of charge carriers $(N)$ versus GN content in the PVC/GN nanocomposites.

entanglements to PVC matrix. The PVC matrix is absorbed and/or coated into the galleries of GN, and the presence of GN in the interfacial regimes between PVC particles shows the nanocomposites without distinguishing individual phases and forming a network of conductive paths. On the other hand, in continuous phase of composites, GN is bonded and entangled well among particles, and bonded particles show uniform layered structure. The uniformity in the continuous phase of composites yield a higher electrical conductivity as confirmed later in this paper.

Figure 1(d) shows the EDX pattern recorded for GN nanoparticles. The strong peaks for graphite and nickel were noted in the spectrum. No other impurities such as sulfur and nitrogen were detected confirming the high purity of graphite nanosheets. The elemental analysis for graphite and nickel was shown to have an average atomic percentages for $\mathrm{G}$ : Ni as $80: 20$, consistent with the elemental stoichiometric ratio of starting materials.

3.2. Static Electrical Properties. Electrical conductivity is the most sensitive method to monitor the continuity of the conductive filler phase in the entire host polymer matrix [20, 21]. Static electrical conductivity of green PVC and PVC/GN nanocomposites at room temperature of about $25^{\circ} \mathrm{C}$ is depicted in Figure 2. Green PVC is electrically nonconductive with a volume conductivity of less than $10^{-16} \mathrm{~S} / \mathrm{cm}$ in dry state at room temperature $[2,3]$. It is observed that the electrical conductivity increases with increasing GN concentration into composites. Higher content of PVC insulating matrix can block the electrically conductive path. The increase in the conductivity of the composite is attributed to the formation of graphite-nickel clusters [1,2]. With increasing the GN content in composite, electrical conductivity increases continuously, this depends upon the conductive path or contact distance between conductive particles $[1,2]$. At higher content of GN, every GN particle is connected to neighboring GN particle that forms surface-tosurface interaction. Therefore, GN in the composite forms a full conductive network because GN particles have ability to tangle with each other owing to their porous structure $[4,7]$.

It is interesting to note that the low percolation threshold in the PVC nanocomposites indicates that the GN nanoparticles have maintained their large aspect ratios during processing and form an electrically conductive network throughout the PVC matrix as confirmed by SEM images in Figures 1(b) and 1(c). The percolation threshold of PVC/GN nanocomposites is $1 \mathrm{wt} \%$. The percolation threshold for the electrical conductivity strongly depends on the geometry and high aspect ratio of the conducting fillers [8]. Fillers with elongated geometry such as fibers or sheets can be used to achieve very low percolation threshold value, due to the fact that fibers or sheets with higher aspect ratios have great advantage over spherical or elliptical fillers in forming conducting networks in polymer matrices $[7,8]$. This advantage in forming conducting network can be explained by excluded volume theory. The excluded volume of an object is defined as a volume around an object into which the center of another similar object is not allowed if overlapping of the two objects is to be avoided. The more extreme the geometry of the filler particle, the larger its excluded volume [11-14]. The larger the excluded volume, the lower the percolation threshold. These high-aspectratio sheets possess great advantages in forming conducting network in polymer matrix, leading to a lower percolation threshold and linear increasing conductivity.

Again, to confirm the above facts on the effect of GN on conductivity, we measured the carriers mobility $(\mu)$ and number of charge carriers per unit volume $(N)$ as shown in Figure 2. It is clearly seen that both $\mu$ and $N$ increase with increasing GN concentration into composites. The increase of both $\mu$ and $N$ is due to the combined effect of an increase in the dimension density of conductive phases and a decrease in the interparticle gaps among conductive sites. This is strong clue that the GN nanoparticles act as carrier's reservoir into PVC matrix, and improving the conductive pathway entire composites. This result leads to the facilities of charge carriers diffusion within PVC matrix, and thus the electrical conductivity increases.

In order to confirm the above facts, we estimated the density of interface states (DIS) at the grain boundary determined by using the following [15]:

$$
\text { DIS }=\left(\frac{2 \varepsilon N E_{a}}{q}\right)^{1 / 2}
$$


Once the concentration of charge carriers $(N)$ and activation energy $\left(E_{a}\right)$ are known, the gap width $(\mathrm{GW})$ among conductive sites was determined by the following:

$$
\mathrm{GW}=\frac{N}{\mathrm{DIS}}
$$

The computed values of DIS and GW as a function of GN content is depicted in Figure 2. It is clear that DIS increases while GW decreases with increasing GN content into composites. This is ascribed to the fact that the inclusion of GN content into composites enhances the number of elastically effective chains and interfacial bonding among filler and matrix.

However, the relation between composite conductivity and conducting filler contents in the vicinity of the percolation threshold can be described by a simple power law $[6,8]$ :

$$
\sigma_{c}=\sigma_{f}\left(\phi_{f}-\phi_{c}\right)^{\beta}
$$

where $\sigma_{c}$ is the composite conductivity, $\sigma_{f}$ is the conductivity of conductive filler, $\phi_{f}$ is the volume fraction of conductive filler, $\phi_{c}$ is the percolation threshold, and $\beta$ is the critical exponent. For lattice in three dimensions, $\beta$ usually lies between 1.65 and 2.0, accepted as a universal value $[5,9]$.

The computed value of the critical exponent $(\beta)$ for $\mathrm{PVC/GN}$ nanocomposites is 3.12 , and this value is much higher than the universal one, indicating the nonuniversal transport behavior of the PVC/GN nanocomposites [15-18]. It has been demonstrated that very high values of critical exponents tend to occur when the conducting particles have extreme geometries $[5,6]$. Presence of tunneling conduction can also lead to nonuniversal critical exponent $[10,11]$. The graphite nanosheets used here possess an average aspect ratio of as high as about 400. Such an extreme geometry might be a factor contributable to the high critical exponent. Thus, tunneling through the insulating PVC barriers is expected leading to nonuniversal transport properties of the conducting nanocomposites $[7,8]$.

3.3. PTCR Thermistors and Transport Mechanism. The temperature dependence of conductivity was examined, as a tool for understanding the mechanism of charge carrier's transport in the PVC/GN nanocomposites and to get a possibility of the application of PVC/GN nanocomposites as PTCR thermistors devices. The temperature dependence of electrical conductivity of PVC/GN nanocomposites is depicted in Figure 3. It is seen that a transition temperature of PTCR curves systematically moved to higher temperatures as the GN content was increased. With increasing GN content, the transition temperature shifted higher. This behavior indicates that more volume expansion of the polymer, allowed for by the increased temperature, was required to pull the conducting particles apart [21]. Increasing the GN content well tended to further decrease the room temperature resistivity and the magnitude in the PTC effect. This strong clue indicates that the inclusion of GN content enhances the thermal stability and skeleton

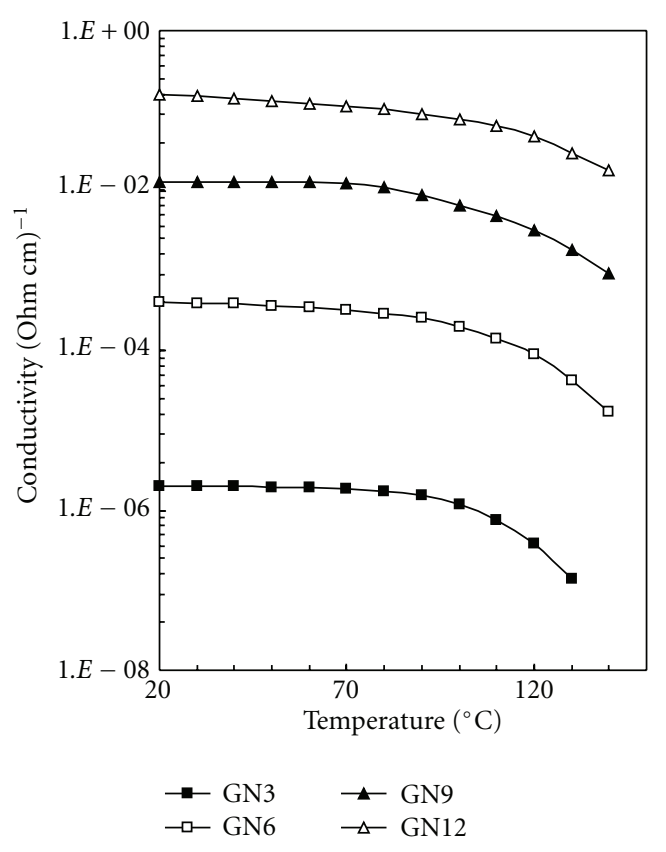

FIGURE 3: Electrical conductivity temperature dependence of PVC/GN nanocomposites.

molecular structure of nanocomposites. Clearly, at relatively low temperature, the conductivity slightly decreases up to certain temperature (i.e., so-called critical or percolation temperature) after which the conductivity quickly increases depending on GN content into composites. There are two main reasons evoked for these phenomena. First, as potential barrier increases proportionally with temperature, electrical resistivity increases very quickly as it depends exponentially on potential barrier. Second, at high temperature, the thermal expansion of polymer increases and the intermolecular distance among conductive segments increases, which leads to the scattering of charge carriers and thereof the conductivity decreases (i.e., resistivity increases).

The electrical conductivity $(\sigma)$ is related to the activation energy $\left(E_{a}\right)$ by $[17,18]$

$$
\sigma=A \exp \left(-\frac{E_{a}}{K_{B} T}\right)
$$

where $A$ is a constant, $K_{B}$ is the Boltzmann constant, and $T$ is the Kelvin temperature.

The activation energy of the nanocomposites was calculated from the slope of $\ln \sigma$ versus $1 / T$ curve, and the data is depicted in Figure 4. It is observed that the activation energy decreases with an increase of GN amount into the nanocomposites. This could occur due to the increased charge carrier concentration, which leads to an increase of the localized state density in the band gap $[1,16]$.

Mott's Variable-Range-Hopping (VRH) model is extensively used to analyze the temperature dependence of $\mathrm{dc}$ conductivity in conducting polymer composites and for 


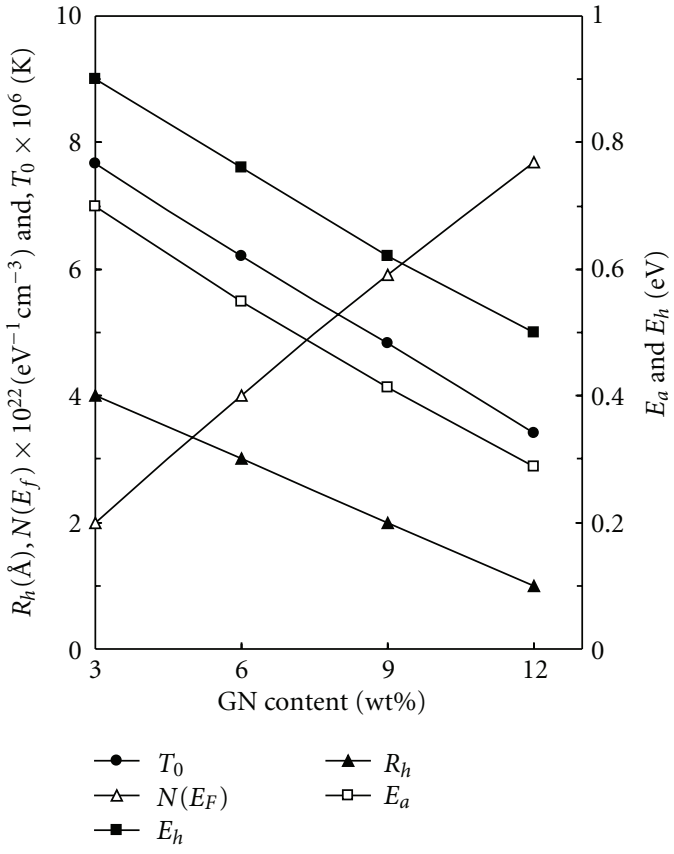

Figure 4: Computed values of $R_{h}, N\left(E_{f}\right), T_{0}, E_{a}$, and $E_{h}$ for PVC/GN nanocomposites.

the three-dimensional hopping mechanism, which is expressed as $[19,20]$

$$
\begin{gathered}
\sigma=\sigma_{0} \exp \left(-\frac{T_{0}}{T}\right)^{1 / 4}, \\
T_{0}=\frac{16}{K_{B} N\left(E_{f}\right) \gamma^{3}},
\end{gathered}
$$

where $N\left(E_{f}\right)$ is the density of states at the Fermi level, $T_{0}$ is the Mott's temperature, and $\gamma$ is the localization length and is taken to be $3 \AA[7,8]$.

The average hopping distance $\left(R_{h}\right)$ between two localized states and the hoping energy $\left(E_{h}\right)$ is given by the following $[3,13]$ :

$$
\begin{gathered}
R_{h}=\left(\frac{3}{8}\right)\left(\frac{T_{0}}{T}\right)^{1 / 4}, \\
E_{h}=\left(\frac{1}{4}\right) K_{B} T\left(\frac{T_{0}}{T}\right)^{1 / 4} .
\end{gathered}
$$

The computed value of $T_{0}, N\left(E_{f}\right), R_{h}$, and $E_{h}$ as a function of GN content into composites is depicted in Figure 4. It is evident that $T_{0}, N\left(E_{f}\right), R_{h}$, and $E_{h}$ decrease as the GN content increases into composites. The observed values of $T_{0}, N\left(E_{f}\right), R_{h}$, and $E_{h}$ point to an effective energy separation between the localized states $[16,19]$. It is worth mentioning that the conductivity values and estimated Mott parameters indicate a good interaction between PVC polymer matrix and GN nanoparticles. The interaction among PVC and GN fillers could be explained by the strong molecular interaction between fillers and matrix. Finally, in Figure 4, it is interesting to note that the value of $E_{a}$ is different from

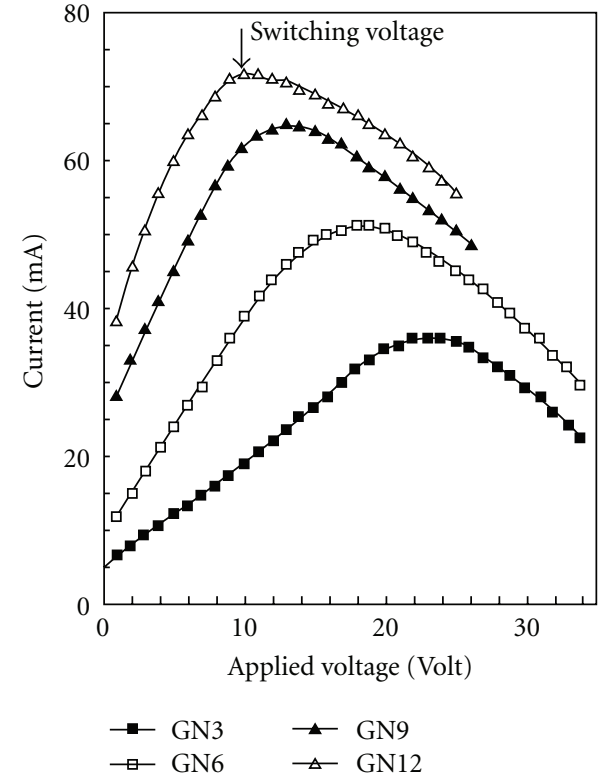

FIGURE 5: Current-voltage dependence of PVC/GN nanocomposites.

the value of $E_{h}$. This is strong evidence that the conduction mechanism of PVC/GN nanocomposite is governed by tunneling mechanism as confirmed above $[9,16]$.

\subsection{Voltage-Current Relationship and Self-Heating Behavior.} The variation of current $(I)$ with applied voltage $(V)$ for PVC/GN nanocomposites is depicted in Figure 5. The increase of GN content shifted the $V-I$ curves toward a low electric field and a high current. This indicates that migration of curves will cause a lower nonlinearity [11]. Clearly the $I-V$ characteristics are linear at low applied voltage without any remarkable change of the sample temperature, indicating the tunneling of electrons on the application of voltage $[2,14]$. Increasing the electric field above, a certain value depends on GN contents, leading to an increase in the Joule heating effect and consequently an increase in the bulk sample temperature. Therefore, $I-V$ characteristics deviate from linear (Ohmic) to nonlinear (non-Ohmic) behavior. By increasing the electric field to a certain value (i.e., switching voltage, see Figure 5), which depends on GN contents, the current decreases showing negative resistance (i.e., switching effect). The negative resistance is attributed to two reasons. First, at high applied voltage the Joule heating takes place and the temperature of the bulk composites increases. This results in an increase in the interparticles distance among conductive sites and the transport of charge carriers decreases. Second, the decrease of current is clearly generated by a repulsive force among positive and negative charges at high applied potential in the PVC matrix [4].

The dependence of surface temperature $(T)$ on applied voltage for PVC/GN nanocomposites is depicted in Figure 6. It is seen that at low voltage the surface temperature slightly increased. With increasing applied voltage, the surface temperature increases; this is due to the Joule or selfheating effect. It is worthily to note that the temperature 


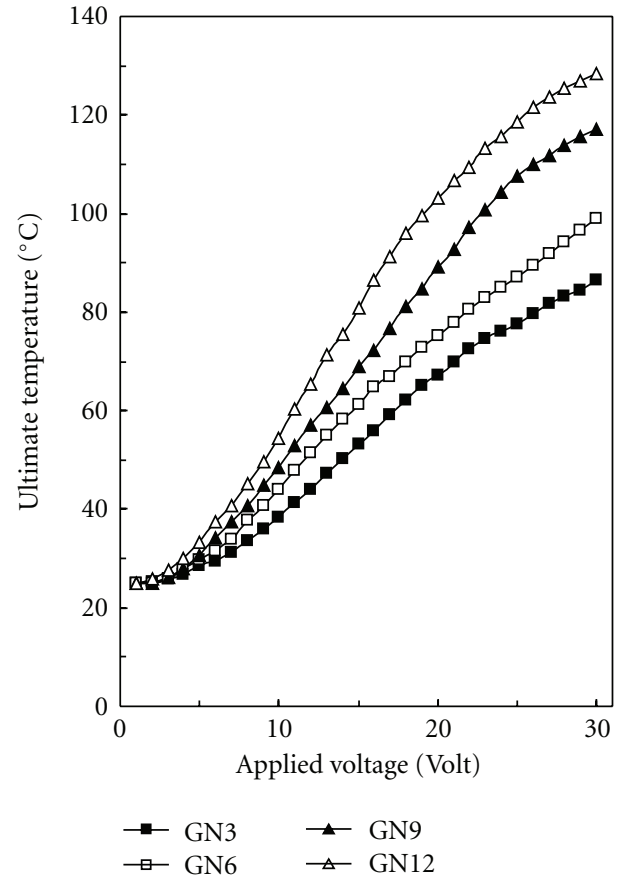

Figure 6: Dependence of ultimate temperature on applied voltage of PVC/GN nanocomposites.



Figure 7: Temperature-time dependence of GN12 sample under applied voltage on and off.

increases with increasing GN content in the nanocomposites. Another argument indicates that the thermal stability of the nanocomposites is enhanced by increasing GN loading level into PVC matrix.

The ultimate surface temperature ( $T$ ) of GN12 sample as a function of time $(t)$ at constant applied power on (22 volts) and off is depicted in Figure 7. In Figure 7, the $(T-t)$ curve can be divided into three regimes.

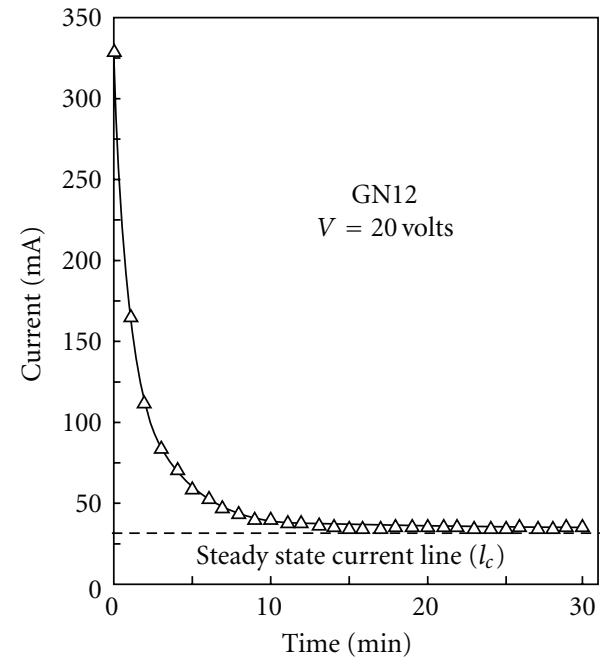

FIGURE 8: Current-time dependence of GN12 sample under applied voltage 20 volts.

Regime I. The applied power on and the temperature increases exponentially with time. In this regime the $(T-t)$ curve can be described by the exponential growth function as follows:

$$
T(t)-T_{1}=\left(T_{2}-T_{1}\right)\left\lfloor 1-\exp \left(-\frac{t}{\tau_{g}}\right)\right\rfloor,
$$

where $T_{1}$ and $T_{2}$ are the ambient and maximum temperature, respectively, and $\tau_{\mathrm{g}}$ is the growth time constant, calculated at $t=\tau_{\mathrm{g}}$.

Regime II. The temperature level off to a steady state value (i.e., equilibrium regime) and based on energy balance law the heat gain by applied working power is equal to heat loss by radiation and convection per area per degree per second $\left(h_{r}\right)$ is given by

$$
h_{r}=\frac{V I_{c}}{T_{2}-T_{1}},
$$

where $V$ is the applied potential and $I_{c}$ is the steady state current after applied potential.

The variation of current with time for GN12 sample after applied potential is depicted in Figure 8. In Figure 8, it is seen that the current decreases with time and then levels off to a steady value and $(I-t)$ curve can be described by the following:

$$
I(t)-I_{0}=\left(I_{c}-I_{0}\right) \exp \left(\frac{t}{\tau_{i}}\right),
$$

where $I_{0}$ and $I_{c}$ are the initial and steady state current, respectively, and $\tau_{i}$ is a decay time constant describing the decay rates of current.

Regime III. In the power off region the $(T-t)$ curve can be described by the exponential decay equation:

$$
T(t)-T_{1}=\left(T_{2}-T_{1}\right) \exp \left(\frac{t}{\tau_{d}}\right),
$$


where $\tau_{d}$ is the decay time constant and is related to the heat transfer from the sample to the surrounding which depends on the GN content in composite.

In this regime (i.e., power off), if the thermistor has a uniform temperature during cooling, the following equation is valid for the cooling, of thermistor in the time interval $d t$ and according to the Newton's law of cooling $[4,5]$ :

$$
C_{p \text { (Newton's })}=\frac{h_{r}\left(T_{2}-T_{1}\right) d t}{m d T} .
$$

The solution of this equation for any value of time $(t)$ is given by (8). From (8) and (9) we obtain $C_{p \text { (Newton's) }}$ in the form $[4,5]$

$$
C_{p(\text { Newton's })}=\left(\frac{h_{r} \tau_{d}}{m}\right)\left(1-\exp \left(-\frac{t}{\tau_{d}}\right)\right) .
$$

For the sake of using these nanocomposites in industrial applications, it is useful to define the whole amount of heat transfer from the composites using the conservation law of energy. We consider that for an applied voltage $(V)$ on composites at time $t=0$. The self-heating effect causes the sample temperature to increase from $T(t)$ to $T(t+d t)$ at a time interval from $t$ to $t+d t$, which produce an electrical energy (i.e., Joule heat energy). According to the energy conservation approach one has the following $[20,21]$ :

$$
\begin{aligned}
\int_{t=0}^{t=t_{m}} V I(t) d t= & m C_{p(\text { cons })} \int_{t=0}^{t=t_{m}} \frac{\partial T_{s}(t)}{\partial t} d t \\
& +A h_{r} \int_{t=0}^{t=t_{m}}\left(\left(T(t)-T_{1}\right)\right) d T
\end{aligned}
$$

where $m$ is the mass of the specimen, $A$ is the area of the specimen, and $C_{p(\text { cons })}$ is the specific heat capacity of the composite.

Taking (7)-(12) into (13) and integrating the resultant equation will obtain the following:

$$
\begin{aligned}
V I_{c} t+ & V\left(I_{0}-I_{c}\right) \tau_{i}\left[1-\exp \left(-\frac{t}{\tau_{i}}\right)\right] \\
= & m C_{p(\text { cons })}\left(T_{2}-T_{1}\right)\left[1-\exp \left(-\frac{t}{\tau_{g}}\right)\right] \\
& +h_{r c} A\left(T_{2}-T_{1}\right) t-h_{r c} A\left(T_{2}-T_{1}\right) \tau_{g} \\
& \times\left[1-\exp \left(-\frac{t}{\tau_{g}}\right)\right] .
\end{aligned}
$$

By integrating (10) we get $C_{p c}$ in the form:

$$
\begin{aligned}
C_{p c}= & \left(\frac{1}{m\left(T_{2}-T_{1}\right)}\right)\left[V_{a} t_{m}\left(T_{2}-T_{1}\right)\right] \\
& +\ln \left(\frac{t_{m}+t_{0}}{t_{0}}\right)+V_{a} I_{m} t_{m} \\
& -A h_{r}\left(T_{2}-T_{1}\right)\left(t_{m}-\exp \left(-\frac{t}{\tau_{g}}\right)\right) .
\end{aligned}
$$

The computed values of $\tau_{g}, \tau_{d}, \tau_{i}, h_{r}, C_{p(\text { Newton's) }}$, and

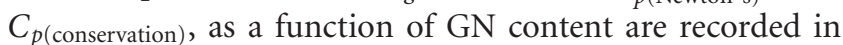

TABLE 1: Some physical properties of PVC/GN nanocomposites.

\begin{tabular}{lcccc}
\hline Physical parameter & GN3 & GN6 & GN9 & GN12 \\
\hline$C_{p}$ (measured) $\left(\mathrm{J} \mathrm{Kg}^{-1} \mathrm{~K}^{-1}\right)$ & 680 & 683 & 688 & 693 \\
$C_{p}$ (Newton's) $\left(\mathrm{J} \mathrm{Kg}^{-1} \mathrm{~K}^{-1}\right)$ & 682 & 685 & 689 & 695 \\
$C_{p}$ (conservation) $\left(\mathrm{J} \mathrm{Kg}^{-1} \mathrm{~K}^{-1}\right)$ & 681 & 684 & 690 & 694 \\
$\tau_{g}(\min )$ & 12.34 & 11.01 & 8.45 & 4.65 \\
$\tau_{d}(\min )$ & 13.43 & 10.21 & 7.78 & 4.23 \\
$\tau_{i}(\min )$ & 11.98 & 7.48 & 6.37 & 3.98 \\
$h_{r}\left(\mathrm{~J} \mathrm{~m}^{-2} \mathrm{~s}^{-1 \circ} \mathrm{C}^{-1}\right)$ & 81 & 74 & 66 & 54 \\
\hline
\end{tabular}

Table 1 . By a close look at Table 1 , it is seen that $\tau_{g}, \tau_{d}$, and $\tau_{i}$ decrease with increasing GN content into composites. This is referred to where the increase of the number of elastically effective chains density increases with increasing GN content into composites. This is strong clue that the inclusion of GN nanoparticles enhances the skeleton internal structure of the PVC matrix, thus rendering it more thermodynamically stable. Also, it is observed that the $h_{r}$ decreases as GN content increases indicating the lower efficiency of heat transfer by radiation and convection into composites. This reflects that the inclusion of GN improves the inner architecture structure and crosslinking density of nanocomposites.

Finally, the measured and computed values of specific heat capacity increase with increasing GN content into nanocomposites. It is interesting to note that the measured and estimated values of specific heat capacity by conservation law of energy and Newton's law of cooling are quite close. This is evidenced in that the inclusion of GN improves the thermal stability and thermal conductivity of the nanocomposite. Monitoring dynamic resistivity (i.e., isothermal resistance relaxation with time) provided additional information about the stability of composites. The isothermal resistance changes with time at constant temperature of $110^{\circ} \mathrm{C}$ for PVC/GN nanocomposites as is depicted in Figure 9. It is clear that the resistance undergoes a sudden increase followed by a decrease in resistance, after which it levels off depending on the GN content into composites. The sudden increase of resistance at the beginning is attributed to a rapid increase of volume expansion of the polymer matrix. It is clear that the time for the resistance to reach the maximum is shorter as the GN content increases in composites. This can be explained where, at low content of GN, the slow diffusion of GN nanoparticles occurs from one grain site to another leading to change the charge distribution which in turn will change the effective carrier density and hence the composite resistivity. In light of the above discussion, we propose that the interfacial bonding and entanglements among PVC moieties increase with the increase of GN contents.

\section{Conclusions}

In this study a new family of electro active polymer nanocomposites thermistors and self-heating based on polyvinyl chloride- (PVC-) reinforced foliated graphite and $\mathrm{Ni}$ nanoparticles (GN) have been successfully fabricated. 


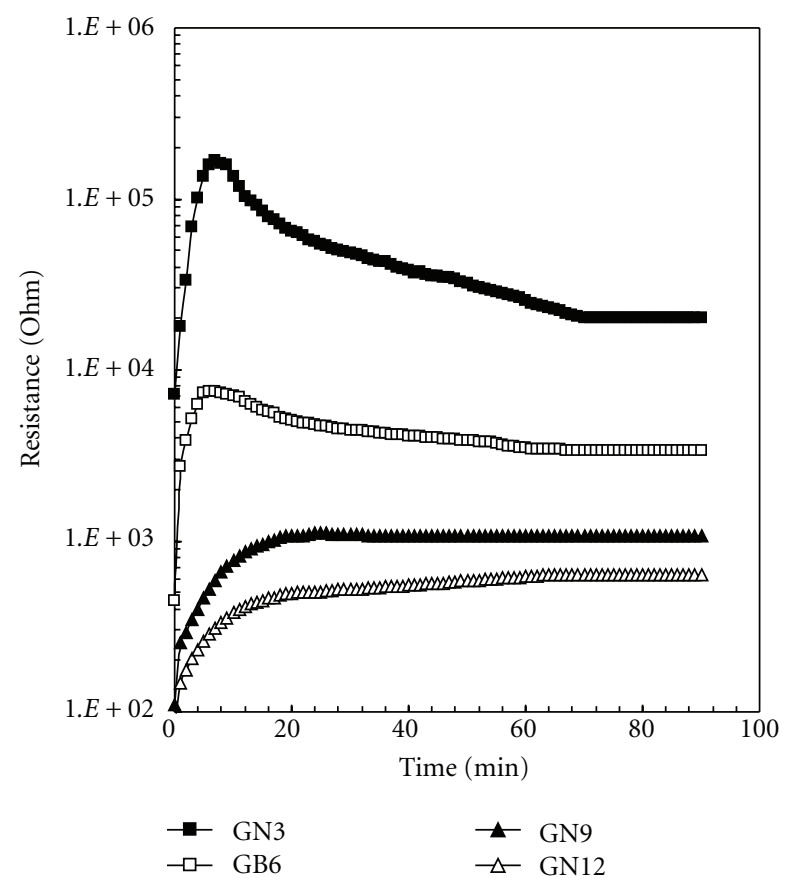

FIgURE 9: Isothermal resistance changes with time at constant temperature of $110^{\circ} \mathrm{C}$ for PVC/GN nanocomposites.

The effect of GN filler loading on the microstructure and electrical properties was studied. Based on the experimental data, the following conclusions can be made.

(1) Scanning electron microscope showed that the inclusion of GN nanoparticles improves the skeleton internal structure of PVC/GN composites.

(2) The electrical conductivity of the PVC/GN nanocomposites showed a transition from an insulator to a semiconductor. The transition could be described by classical percolation theory with a critical exponent of about 3.12. The PVC/GN nanocomposites exhibited the lowest percolation threshold of about $0.7 \mathrm{wt} \%$. This may be attributed to the increased filler form factor in PVC/GN nanocomposites.

(3) The applicability of nanocomposites obtained as PTCR thermistors was examined by monitoring the conductivity as a function on temperature.

(4) The applicability of nanocomposites for selfregulating heater and temperature sensor was tested by displaying the change of current and temperature with applied voltage.

(5) The specific heat capacity and amount of heat transfer by radiation and convection as a function of GN content were estimated by proposed theoretical modeling based on conservation law of energy and Newton's law of cooling.

(6) Finally, we believe that the PVC/GN nanocomposites open a new direction for future application in electronics devices such as PTCR thermistors, self-regulating heater, and temperature sensors.

\section{Acknowledgment}

The authors would like to thank the University of Tabuk, Saudi Arabia, for the financial support of this research.

\section{References}

[1] N. A. Aal, F. El-Tantawy, A. Al-Hajry, and M. Bououdina, "New antistatic charge and electromagnetic shielding effectiveness from conductive epoxy resin/plasticized carbon black composites," Polymer Composites, vol. 29, no. 2, pp. 125-132, 2008.

[2] O. A. Al-Hartomy, F. Al-Solamy, A. A. Al-Ghamdi, M. A. Ibrahim, N. Dishovsky, and F. El-Tantawy, "Pressure sensors based on polyvinyl chloride/graphite/nickel nanocomposites," Journal of Elastomers and Plastics, vol. 43, no. 2, pp. 137-153, 2011.

[3] A. A. Al-Ghamdi, F. El-Tantawy, N. Abdel Aal, E. H. ElMossalamy, and W. E. Mahmoud, "Stability of new electrostatic discharge protection and electromagnetic wave shielding effectiveness from poly(vinyl chloride)/graphite/nickel nanoconducting composites," Polymer Degradation and Stability, vol. 94, no. 6, pp. 980-986, 2009.

[4] F. El-Tantawy and N. Dishovsky, "Novel V-shaped negative temperature coefficient of conductivity thermistors and electromagnetic interference shielding effectiveness from butyl rubber-loaded boron carbide ceramic composites," Journal of Applied Polymer Science, vol. 91, no. 5, pp. 2756-2770, 2004.

[5] G. Chen, W. Weng, D. Wu, and C. Wu, "PMMA/graphite nanosheets composite and its conducting properties," European Polymer Journal, vol. 39, no. 12, pp. 2329-2335, 2003.

[6] J. Lu, X. Chen, W. Lu, and G. Chen, "The piezoresistive behaviors of polyethylene/foliated graphite nanocomposites," European Polymer Journal, vol. 42, no. 5, pp. 1015-1021, 2006.

[7] F. El-Tantawy, "Plasticized/graphite reinforced phenolic resin composites and their application potential," Journal of Applied Polymer Science, vol. 104, no. 2, pp. 697-709, 2007.

[8] Z. Todorova, N. Dishovsky, R. Dimitrov, and F. El-Tantawy, "Investigation of electrical properties of elastomer composites filled with $\mathrm{TiB}_{2}$," Journal of Elastomers and Plastics, vol. 39, no. 1, pp. 69-80, 2007.

[9] G. Chen, W. Weng, D. Wu et al., "Preparation and characterization of graphite nanosheets from ultrasonic powdering technique," Carbon, vol. 42, no. 4, pp. 753-759, 2004.

[10] N. C. Das, D. Khastgir, T. K. Chaki, and A. Chakraborty, "Electromagnetic interference shielding effectiveness of carbon black and carbon fibre filled EVA and NR based composites," Composites Part A, vol. 31, no. 10, pp. 1069-1081, 2000.

[11] X. S. Du, M. Xiao, and Y. Z. Meng, "Facile synthesis of highly conductive polyaniline/graphite nanocomposites," European Polymer Journal, vol. 40, no. 7, pp. 1489-1493, 2004.

[12] S. E. Bourdo and T. Viswanathan, "Graphite/Polyaniline (GP) composites: synthesis and characterization," Carbon, vol. 43, no. 14, pp. 2983-2988, 2005.

[13] S. Shekhar, V. Prasad, and S. V. Subramanyam, "Transport properties of conducting amorphous carbon-poly(vinyl chloride) composite," Carbon, vol. 44, no. 2, pp. 334-340, 2006.

[14] W. Luheng, D. Tianhuai, and W. Peng, "Effects of conductive phase content on critical pressure of carbon black filled silicone rubber composite," Sensors and Actuators, A, vol. 135, no. 2, pp. 587-592, 2007. 
[15] W. Lu, H. Lin, D. Wu, and G. Chen, "Unsaturated polyester resin/graphite nanosheet conducting composites with a low percolation threshold," Polymer, vol. 47, no. 12, pp. 4440 4444, 2006.

[16] M. N. Kalasad, M. A. Gadyal, R. K. Hiremath et al., "Synthesis and characterization of polyaniline rubber composites," Composites Science and Technology, vol. 68, no. 7-8, pp. 1787-1793, 2008.

[17] S. Qu and S. C. Wong, "Piezoresistive behavior of polymer reinforced by expanded graphite," Composites Science and Technology, vol. 67, no. 2, pp. 231-237, 2007.

[18] W. Weng, G. Chen, and D. Wu, "Transport properties of electrically conducting nylon 6/foliated graphite nanocomposites," Polymer, vol. 46, no. 16, pp. 6250-6257, 2005.

[19] G. M. Gouda and C. L. Nagendra, "Structural and electrical properties of mixed oxides of manganese and vanadium: a new semiconductor oxide thermistor material," Sensors and Actuators, A, vol. 155, no. 2, pp. 263-271, 2009.

[20] S. Isaji, Y. Bin, and M. Matsuo, "Electrical conductivity and self-temperature-control heating properties of carbon nanotubes filled polyethylene films," Polymer, vol. 50, no. 4, pp. 1046-1053, 2009.

[21] Y. Song and Q. Zheng, "Electric self-heating behavior of acetylene carbon black filled high-density polyethylene composites," Polymer International, vol. 53, no. 10, pp. 1517-1522, 2004. 



The Scientific World Journal

Submit your manuscripts at

http://www.hindawi.com

\section{World Journal}

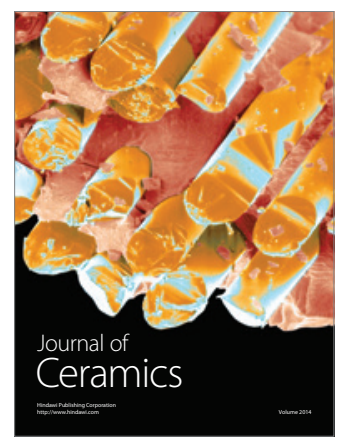


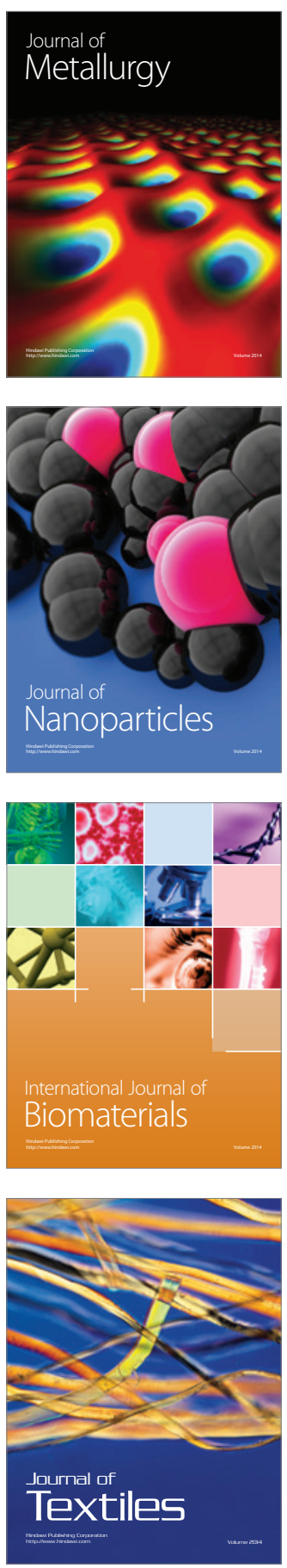